\title{
Towards an In Silico Approach to Personalized Pharmacokinetics
}

\author{
Akihiko Konagaya \\ Tokyo Institute of Technology, \\ Japan
}

\section{Introduction}

The human genome sequence project has made a great impact on medical science and drug discovery (Collins et al., 2003). The rapid progress of genome sequencing technologies enables us to study personal genome sequences with reasonable costs (Mitchelson, 2007). It is now widely believed that personal genome information will be one of the most important biomedical contributions to personalized medicine, that is, medical and health care based on individual genetics (Angrist, 2007). Personalized medicine has opened the doors to new and emerging technologies in genome drug discovery, including pharmacogenetics, pharmacokinetics, and pharmacodynamics, to name but a few. Pharmacogenetics investigates genetic effects in drug metabolic enzymes and drug transporters for drug efficacy (Pirmohamed, 2011). Pharmacokinetics and dynamics (PKPD) focus on the area under plasma concentration time curve (AUC) of drugs, one of the important indices to check the drug effects in the human body, especially for preventing adverse side effects (Gabrielsson et al., 2009).

Although both pharmacogenetics and PKPD have revealed the association between genetic mutations and drug efficacy, their accomplishments are not sufficient yet for clinical purpose, especially with respect to prediction performance (Pirmohamed, 2011). Why are the associations between genetic mutations and drug efficacy so vague? Why can pharmacokinetic models not predict drug metabolism correctly? Why does model parameter fitting not work well in prediction tasks? These questions motivated us to initiate the study of personalized pharmacokinetics, the opposite side of conventional pharmacokinetics, that is, population pharmacokinetics (Willmann et al., 1994).

The basic approach to personalized pharmacokinetics is the breakdown of the pharmacokinetic problem into the dynamics of molecular interactions between drug metabolic enzymes and drug metabolites. In this approach, the molecular interactions and the drug metabolites are represented by ontologies. The key concepts of pharmacokinetics, such as drug metabolic pathways and drug-drug interactions are also represented by the aggregation of molecular interactions and the conflicts of molecular interactions on the same enzymes, respectively. The severities of drug-drug interactions are measured by mathematical simulation models represented by ordinal differential equations corresponding to molecular interactions. 
Personalized pharmacokinetics is one of the in silico studies and trial simulations but differs from population pharmacokinetics in the following sense. The objective is the analysis of the genetic traits of an individual patient rather than the average and distribution of these traits in the patient population. A pharmacokinetic model is constructed from the knowledge base of drug metabolic reactions according to an individual regimen, not from a statistical analysis or machine learning applied to population data. The model is used for the estimation of parameter distribution reproducing the observed clinical data from the viewpoint of an inverse problem.

In order to achieve the above goals, we have developed a prototype system for personalized pharmacokinetics, which consists of the Drug Interaction Ontology, the inference programs for drug-drug interaction detection and generation of metabolic pathways and models, and the pharmacokinetic numerical simulation engine with virtual patient population convergence facility. The Drug Interaction Ontology is a kind of knowledge representation with regards to drug metabolism (Yoshikawa et al., 2004). It consists of two vocabulary hierarchies in terms of process and continuants like the SNAP-SPAN ontology, that is, a combination of a purely spatial ontology supporting snapshot views of the world at successive instants of time and a purely spatiotemporal ontology of change and process (Grenon\&Smith, 2004a). The process vocabulary hierarchy defines the dynamics of drug metabolism. The continuant vocabulary hierarchy defines categories of bio-chemical molecules related to drug metabolism. A drug metabolism knowledge base is developed on the Drug Interaction Ontology as a collection of anonymous objects representing drug metabolic reactions and their aggregation, that is, drug metabolic pathways. The Drug Interaction Ontology is represented by OWL-DL. However, the logic programming language Prolog is used for drug-drug interaction detection and generation of metabolic pathways and pharmacokinetic numerical simulation models.

The inference programs compensate the lack of knowledge in the form of anonymous objects in the drug metabolic pathway knowledge base. The inference programs dynamically generate anonymous instances, such as drug metabolic pathways and detect the occurrences of drug-drug interactions, when a patient's regimens are given. The automatic generation of drug metabolic pathways is indispensable for multiple drug regimens since the total number of drug metabolic pathways becomes the number of all possible combinations of drugs, which are meaningless to be provided in the drug metabolic knowledge base in advance. Instead, the knowledge base consists of a collection of primitive drug metabolic reactions and inference programs to generate drug metabolic pathways of multiple-drug regimens. The drug-drug interaction detector detects the occurrences of drugdrug interactions in the generated drug metabolic pathways and then dynamically adds the occurrences as hypothetical assertions in the drug metabolic pathway knowledge base, mapping the assertions on process and continuant vocabulary hierarchies. The mapping gives useful background information to validate the assertions. In addition, the mapping is also helpful to generate a pharmacokinetic numerical simulation model when choosing an appropriate mathematical equation corresponding to competitive and non-competitive enzymatic inhibition.

The virtual patient population convergence is a concept for solving an inverse problem. The objective is to estimate multiple sets of parameters reproducing the personal clinical datum of an individual patient. The inverse problem approach is very different from conventional 
population pharmacokinetics that tries to estimate the average and diversity of a patient population. In our approach, only the clinical data of an individual patient are necessary. This enables us to analyze an "outlier" of the population. We strongly believe that the analysis of such outliers is more important than the analysis of average behaviors for practical personalized pharmacokinetics.

In order to demonstrate the effectiveness of our approach, we applied the prototype system to the pharmacokinetic studies of anti-cancer drug irinotecan with a whole body pharmacokinetic model with regards to the hepatic and renal excretions of five major irinotecan metabolites: CPT-11 (irinotecan), APC1, NPC2, SN-38 (active metabolite of irinotecan), and SN-38G (SN-38glucuronide). Firstly, we investigated how ketoconazole affects the irinotecan metabolite blood concentrations with the pharmacokinetic model in (Arikuma et al., 2008). Ketoconazole inhibits CYP3A4, one of the drug metabolic enzymes of irinotecan. The numerical simulation analysis revealed an interesting behavior of the drug metabolism, which is difficult to expect from pathway-level analysis. Then, we investigated how the UGT1A1*28/*28 mutation affects SN-38 blood concentration in the Arikuma model. UGT1A1*28/*28, also known as Gilbert's syndrome, is one of the important mutations that may cause severe side-effects when using irinotecan (Tukey et al, 2002). The simulation result suggests how the expression level of UGT1A1 affects the metabolite concentration of SN-38. Lastly, we investigated kinetic parameters reproducing a bile-duct cancer patient who showed a metabolite excretion profile that was completely different from other cancer patients. This is a typical example of an inverse problem. The result indicates that the analysis of the solution space of the inverse problem is a key to understanding the peculiar behavior of an outlier like this bile-duct cancer patient.

The organization of this chapter is as follows. Firstly, we describe our motivation and background of studying personal pharmacokinetics in Section 2. Sections 3 describes the prototype system which consists of the Drug Interaction Ontology, inference programs for pathway (model) generation and drug-drug interaction detection, and numerical simulation engine with the facility of virtual patient population convergence. Section 4 introduces three case studies of irinotecan pharmacokinetics, drug-drug interaction of irinotecan and ketoconazole, mutation analysis of drug metabolic enzyme UGT1A1, and an inverse problem analysis of a bile-duct cancer patient with an external bile drain. Section 5 discusses the controversial points of this work. Finally, section 6 gives the conclusions of our study.

\section{Personalized pharmacokinetics}

The role of in silico prediction of drug interactions at the pathway level is becoming more and more important for personalized medicine. Multiple-drug regimens exemplify the need for the computer-assisted prediction of drug interactions, which may be different from one patient to another. Multiple-drug regimens are commonly prescribed for elderly patients suffering from more than one disease. However, these regimens sometimes cause unexpectedly severe side effects because of the drug interactions or individual differences concerning response to the drugs (Okuda et al., 1998). Therefore, the prediction of drug

1 7-ethyl-10-[4-N-(5-aminopentanoic acid)-1-piperidino] carbonyloxycamptothecin

2 7-ethyl-10-(4-amino-1-piperidino) carbonyloxycamptothecin 
interactions for preventing the side effects is an important issue for these regimens. On the other hand, the information useful for in silico drug interaction prediction has increased very rapidly in recent years. Technological innovations in genomic sciences have produced an enormous amount of bio-molecular information including sequences, structures, and pathways. In order to integrate the bio-molecular information, ontologies are attracting a lot of attention (Baker \& Cheung, 2007; Konagaya, 2006a, 2006b). In addition, pharmacokinetics modeling and simulation are emerging, promising techniques to understand the dynamic behavior of drug metabolic pathways (Tsukamoto et al., 2001;Vossen et al., 2007). To develop personalized pharmacokinetics that can deal with individual drug administration including multiple-drug regimens by integrating the above information and techniques, the following issues must be solved:

- Context dependency of drug-metabolic pathways,

- Treatment of multi-scale events,

- Quantitative evaluation of interactions,

- Automatic generation of simulation models,

- Inverse problem solving of an outlier, and so on.

Drug-metabolic pathways do not exist a priori. They strongly depend on contexts and situations including the administration route, single nucleotide polymorphism (SNP) of drug-response genes, and the administration of multiple drugs and foods. Therefore, a dynamic reconstruction of drug metabolic pathways from primitive molecular events is necessary for drug interaction prediction at the pathway level. Such a reconstruction requires the formal definition of molecular events and the relations among them, i.e., the Drug Interaction Ontology (DIO) (Yoshikawa et al, 2004).

Pathways triggered by drug administration consist of multi-scale events: from the molecular level to the body level, ranging from nanoseconds to hours or days in terms of drug response. For example, drug administration and drug excretion are body-level events, while drug transport and enzymatic reactions are molecular-level events. A comprehensive view from the molecular level to the body level is necessary in order to understand multi-scale events.

Quantitative evaluation plays an essential role to estimate the degree of side effects caused by drug interactions. More than one drug interaction may occur in drug-metabolic pathways from the qualitative reasoning viewpoint. However, not all drug interactions cause side effects because of differences in binding affinity and molecular population. Quantitative simulation models with an in silico drug interaction prediction system must be incorporated to discriminate serious drug interactions from negligible ones. It should also be noted that the total drug metabolism depends on not only kinetic parameters but also physiological parameters such as organ volumes and blood flows. The incorporation of kinetic and physiological parameters is necessary for a realistic simulation model to recapture experimental data.

The automatic generation of mathematical models is necessary to avoid a combinatorial problem of drug metabolic pathways caused by multi-drug regimens and the occurrences of drug-drug interactions. On-demand automatic generation of simulation models also liberates modelers from the tedious editing of complex differential equations. 
In the case of pharmacokinetics, we often encounter an outlier caused by a peculiar patient who shows different clinical data from other patients. In order to analyze such an outlier, inverse problem solving is necessary. Since most pharmacokinetic models are underdetermined, that is, they have dozens of parameters which are difficult to determine by biological experiments or clinical observation, the solution space of inverse problems becomes a multiple-set or a manifold. Finding multiple sets that reproduce the observed clinical data on a pharmacokinetic model is one of the major challenges in numerical simulation. We solved this issue by restricting the search space within physiologically reasonable ranges, and by utilizing intelligent sampling techniques with a Markov-Chain Monte Carlo (MCMC) method and Support Vector Machine (SVM) method in the prototype system.

\section{Prototype system}

The prototype system for personalized pharmacokinetics consists of the Drug Interaction Ontology, the inference programs for drug-drug interaction detection and generation of metabolic pathways and models, and the pharmacokinetic numerical simulation engine with virtual patient population convergence facility (Fig.1).

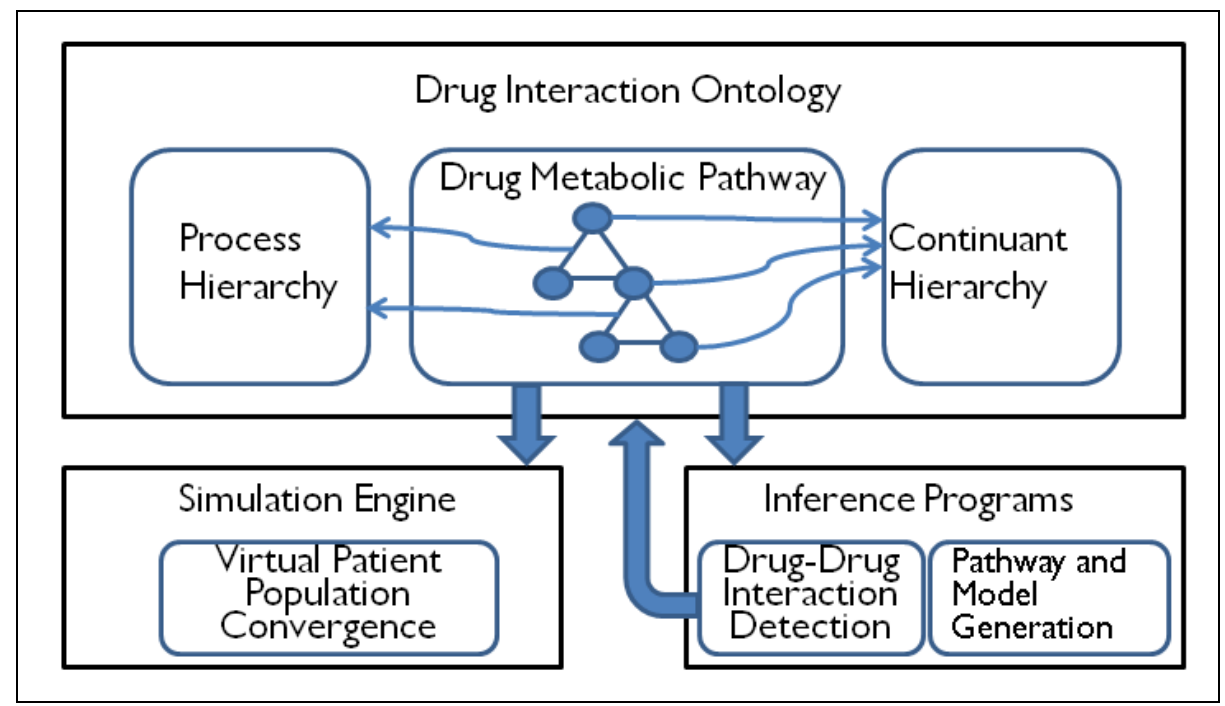

Fig. 1. The Organization of the Prototype System

The prototype system consists of the Drug Interaction Ontology, inference programs and simulation engine. A drug metabolic pathway is represented by an anonymous object whose compounds are linked to terms in the continuant hierarchy, and whose reactions are linked to terms in the process hierarchy. The inference programs generate a metabolic pathway by aggregating primitive reaction objects. After detecting the occurrences of the drug-drug interaction, the generated pathway is translated to a model, that is, a list of ordinal differential equations. The simulation engine estimates a virtual patient population reproducing an observed clinical datum by means of solving an inverse problem with the equations. 


\subsection{Drug Interaction Ontology (DIO)}

The ontological approach in knowledge base design is adopted for resource sharing and the semantic description of molecular events and pathways. Ontology is necessary to define molecular events and pathways in a form that can be shared among computers and human beings. This enables the full use of powerful computational intelligence for dynamic pathway reconstruction in a way that human intelligence can follow and understand. Ontology is also important for establishing interoperability among web resources and thereby to make use of the latest drug reaction information published in the semantic web (Baker\&Cheung, 2007; Berners-Lee\&Hendler, 2001). Public biological ontologies, especially in the field of chemical biology, are now dramatically increasing and have a great potential to develop sustainable knowledge bases for molecular reaction and pathways (Ceusters\&Smith, 2010).

The Drug Interaction Ontology is designed to share the knowledge of drug-drug interaction in both machine and human understandable form. The controlled vocabularies of the Drug Interaction Ontology consist of "process" (Fig. 2) and "continuant" (Fig. 3) as proposed in Basic Formal Ontology (BFO) (Grenon et al., 2004b). Molecular events are asserted in the knowledge base referring the terms of the controlled vocabularies. A drug metabolic pathway is represented by the aggregation of molecular events. This is because an infinite number of terms or classes are required to express all combinations of molecular events. We avoid this problem by treating pathways as anonymous objects deduced from prototype molecular event objects rather than treating them as instances of pathway classes.

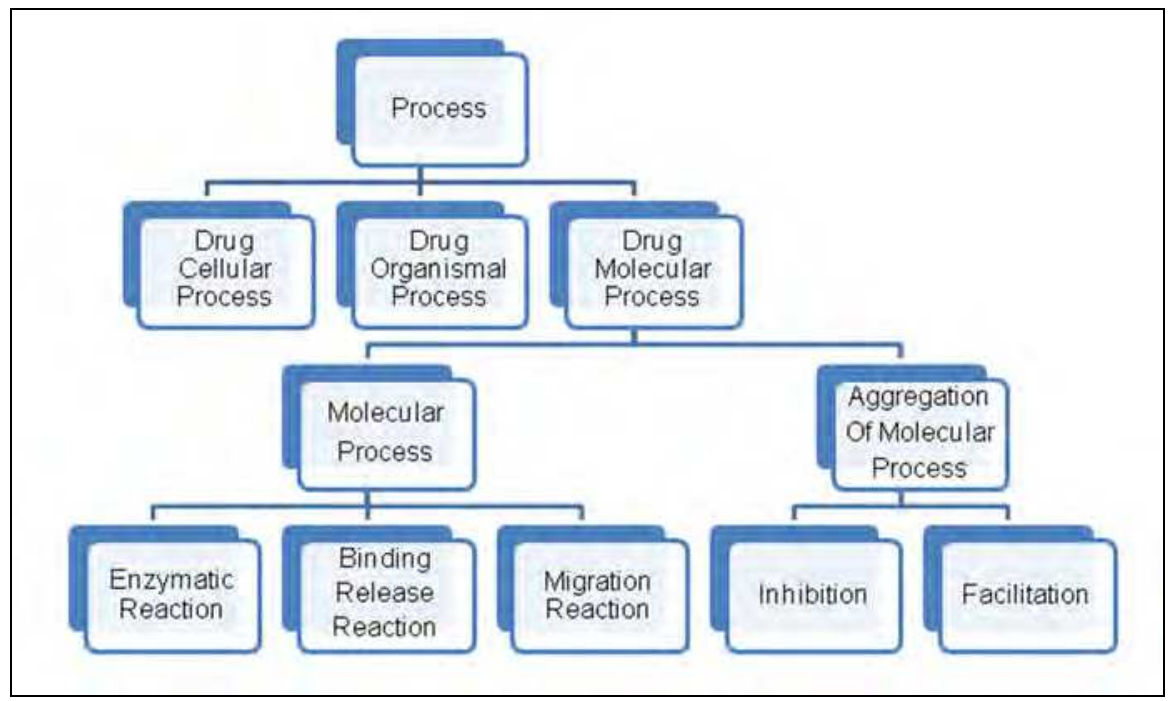

Fig. 2. A Part of Process Vocabulary Hierarchy

Process vocabulary hierarchy defines the terminology of metabolic reactions from molecular level to body level. Note that each term in the process hierarchy may have more than two kinds of anonymous reaction objects or their aggregations categorized into the same term. This facility enables to avoid combinatorial expansion of terms to deal with aggregation of anonymous reaction objects. 


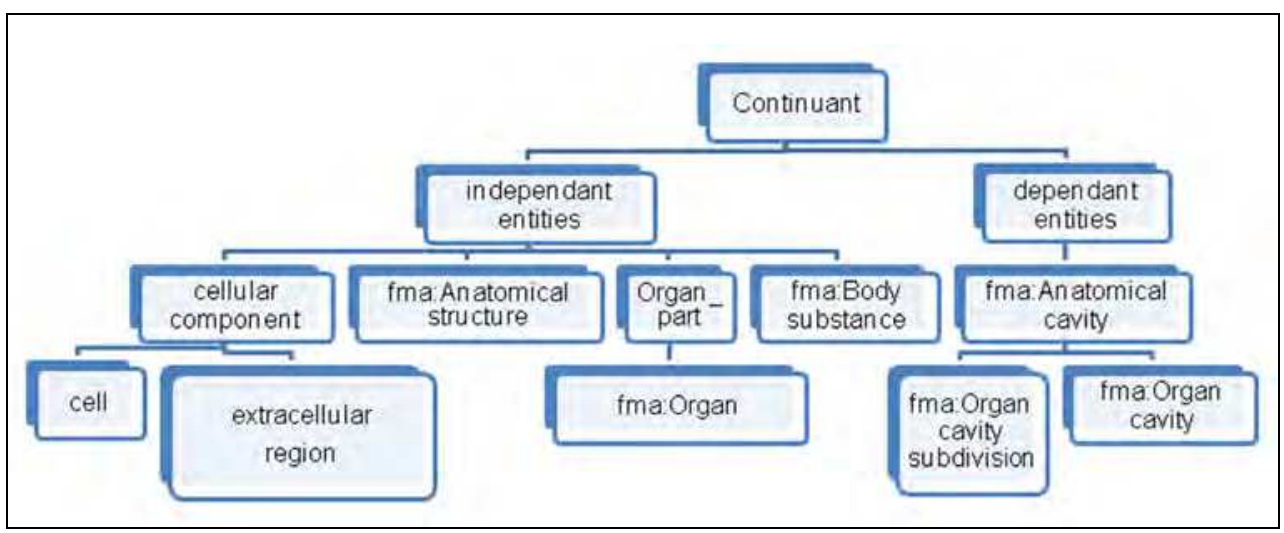

Fig. 3. A Part of Continuant Vocabulary Hierarchy

Continuant vocabulary hierarchy defines the terminology of compounds, cells and organs which contribute the human body. The terminologies imported from reference ontologies are prefixed by identifies such as "fma" and "umls" for the Foundational Model of Anatomy and Unified Medical Language System, respectively.

Ontology provides not only a class hierarchy of controlled vocabulary but also property and relations among terms useful for inferences. The inferences may include qualitative reasoning and numerical simulation, i.e., pathway generation, drug-drug interaction detection, differential equation generation, and numerical simulation as well as reasoning on OWL-DL. Obtained results such as drug interaction candidates can be dynamically mapped on the ontology as hypothetical assertions. This approach is attractive since it can use ontology as background knowledge to interpret the inference results.

The Drug Interaction Ontology (DIO) was written in OWL-DL; the controlled vocabularies of process and continuant were implemented as OWL class hierarchy, and part of attributes, including molecules and organs, were implemented as OWL instances. The molecular event objects were represented by OWL instances and OWL properties. The ontology referred to other taxonomies and ontologies for well-established vocabularies of biochemical terms, anatomical entities, and properties. This enabled the reduction of our ontology construction cost and to concentrate our efforts on the information specific to drug interaction.

\subsection{Automatic generation of drug metabolic pathways}

The drug metabolic pathway, due to its dynamic nature, is difficult to define a priori in the manner as seen in bio-molecular metabolic pathways in the Kyoto Encyclopedia of Genes and Genomics (KEGG) (Kanehisa\&Goto, 2000). Therefore, modularization is necessary for the dynamic reconstruction of pathways that depend on dose conditions. Careful selection of primitive modules is the key to ensuring the soundness of pathway reconstruction. Molecular events, such as molecular transport and enzymatic reactions, are well-formed primitive modules for this purpose. In this chapter, we refer to the primitive modules as "molecular events", and the aggregation of molecular events as "pathways". To avoid redundant pathway branch constructions, which are non-essential for the target drug interactions, we adopt causality-based modularization in which each molecular event is 
defined by the unique relationship between key molecules before and after the event. The triadic relationship <trigger, situator, resultant> is one such causality that can be commonly found in molecular reactions (Yoshikawa et al., 2004). For example, in case of enzymatic reactions, substrates, enzymes and other products correspond to trigger, situator, and resultant, respectively. In the case of molecular transport, extra (intra) cellular molecules, transporters, and intra (extra) cellular molecules correspond to the participants of the triadic relation, respectively. The triadic relationship can be applicable to higher level events like drug dosage and drug excretion, as long as its causality is unique and clear. Figure 4 shows a simple example of pathway reconstruction with two primitive molecular events: an enzymatic reaction in which carboxylesterase (CE) metabolizes irinotecan into SN-38 (7ethyl-10-hydroxycamptothecin) in the liver, and molecular transport in which SN-38 in the liver is transported to the bile by MRP2 (Multidrug resistance-associated protein 2). Two molecular events are connected at the resultant of the enzymatic reaction and the trigger of the molecular transport for passing SN-38 in the liver (SN-38@liver) to the bile.

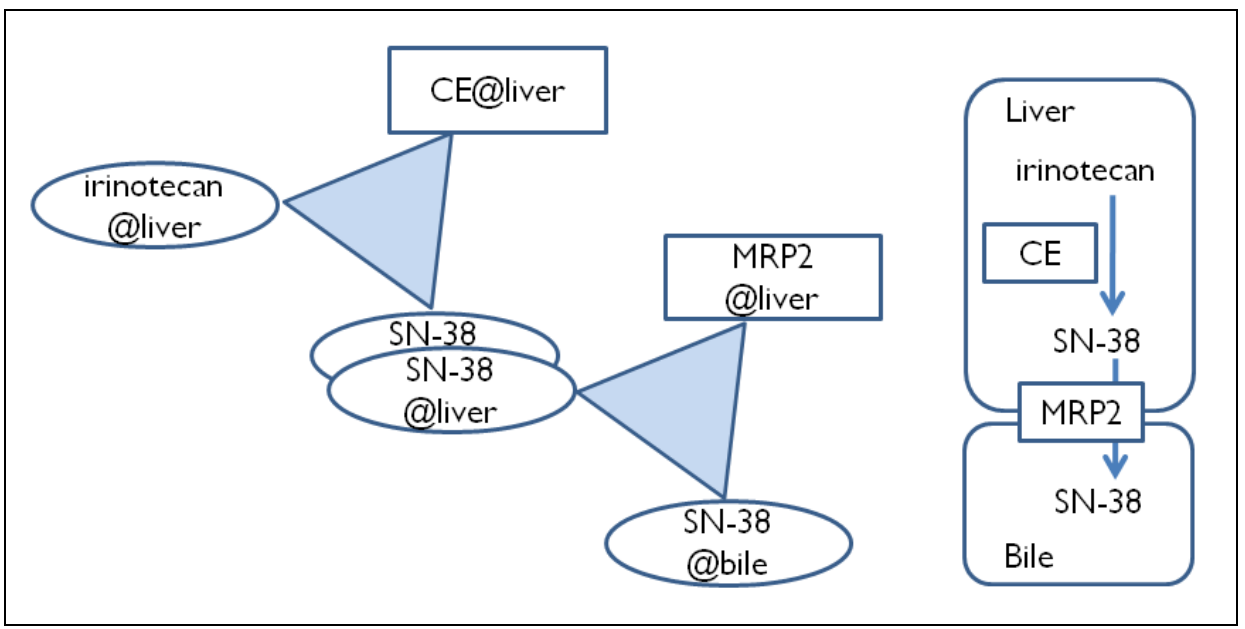

Fig. 4. Reconstruction of Drug Metabolic Pathway from Primitive Reactions

Each primitive metabolic reaction is represented by a triplet of <trigger, situator, resultant>. Metabolic pathway is constructed by aggregation of primitive reactions where a resultant becomes a trigger of the consecutive reaction as like SN-38 for CE and MRP2. Each compound has location information indicated by the @ symbol to deal with migration process across organs such as MRP2.

\subsection{Automatic generation of drug metabolic pharmacokinetic models}

In order to incorporate a quantitative numerical simulation into the prototype system, the following two aspects are considered: a methodology for the automatic conversion from a generated pathway to a quantitative simulation model, and a methodology to solve an inverse problem, that is, virtual patient population convergence. These two methodologies enable us to apply the prototype system to the in silico prediction of individual drug interactions for multiple-drug regimens, assuming that kinetic parameters and the initial enzyme concentration are roughly estimated by individual genetic variations and health 
indices of bio-markers. A simulation model is automatically translated from a drug metabolic pathway generated by the inference programs. The generated pathway is converted to an intermediate model by merging organs and molecular events, respectively, to fit a given simulation model such as a compartment model. Then, a list of ordinal differential equations for the simulation model is generated from the intermediate model by converting the merged events to mathematical expressions.

Figure 5 shows a simulation model automatically generated for the co-administration of irinotecan and ketoconazole. The organs and tissues are integrated into 8 compartments, i.e., blood (including rapidly equilibrating tissues: artery, heart, kidneys, lung, and veins), liver, GI (gastrointestinal consists of the large intestine, small intestine, portal vein, and stomach), adipose tissue, NET (non-eliminating tissue such as skin and muscle), GI lumen, bile lumen,

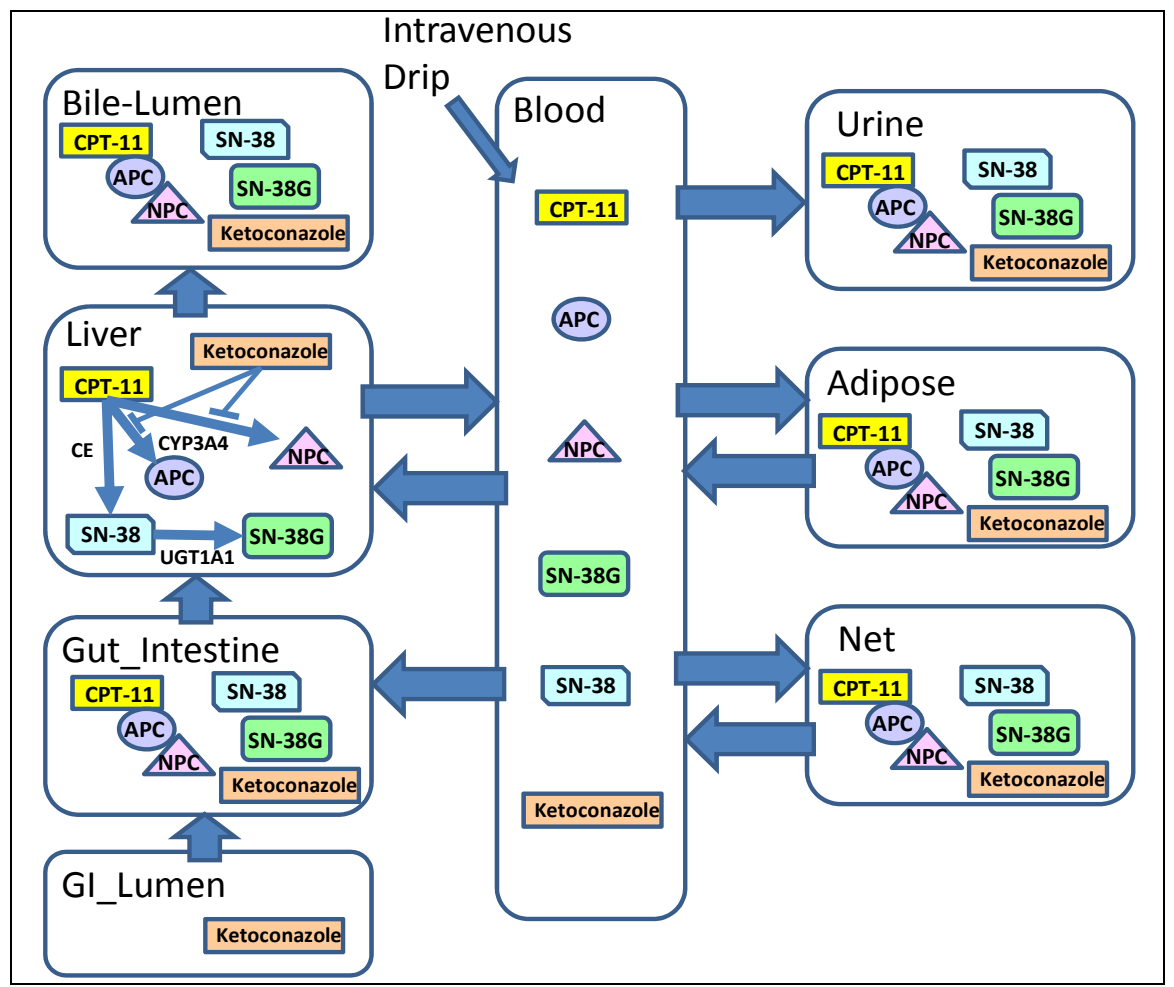

Fig. 5. Generated Pathway Model for Irinotecan and Ketoconazole Co-administration The model consists of 8 compartments: Blood, Liver, Adipose, Gut-Intestine (GI), GI-Lumen, Urine, Bile-Lumen, and Net. Irinotecan is injected directly into the Blood compartment in the way of intravenous drip. The Blood compartment plays a central role to circulate drug metabolites to other compartments. The GI-Lumen and the GI compartments are provided for the oral administration of ketoconazole. The Urine and the Bile-Lumen compartments are provided for the renal and hepatic excretions of irinotecan metabolites. Drug metabolic reactions are occurred in the Liver compartment. The Adipose and the Net compartments are provided for the difference of blood circulation speeds of organs. 
and urine. Michaelis-Menten equations are used for all enzymatic reactions. A competitive Michaelis-Menten inhibition model is used for this simulation as used for midazolam and ketoconazole inhibition by (Chien et al., 2006). In order to increase the predictive performance, a simplified pathway is used for the generation of simulation models from the viewpoint of the trade-off between model complexity and data availability. For example, in the case of irinotecan and ketoconazole metabolisms, reabsorption through small intestine and reactions concerning albumin are omitted due to the lack of information.

The generated simulation models and pharmacokinetic moment parameter values are mapped onto the Drug Interaction Ontology as hypothetical assertions. The simulation models are asserted as aggregations of objects representing terms and parameters in differential equations. Those objects having references to the components of the pathway objects from which the simulation models are generated. The moment parameter values are asserted with the drug interaction objects and the corresponding simulation model for the further analysis. See (Arikuma et al., 2008) for the details of ontology, mathematical models, and their implementations of irinotecan and ketoconazole drug metabolic pathways.

\subsection{Virtual patient population convergence}

Virtual patient population convergence is a concept to find a partial set of virtual patients reproducing an observed clinical datum in some error range with regards to a given mathematical model by means of iteration of numerical simulation and population selection starting from an initial virtual patient population (Fig.6). In general, the solution space of a mathematical model, that is, the virtual patient population reproducing an observed clinical data on the model, might be infinite. In addition, there is a trade-off between the virtual patient sampling time and the precision of the virtual patient population converged to the observed clinical data. Therefore, some kind of criteria with regards to the "goodness" of convergence for virtual patient population must be defined.

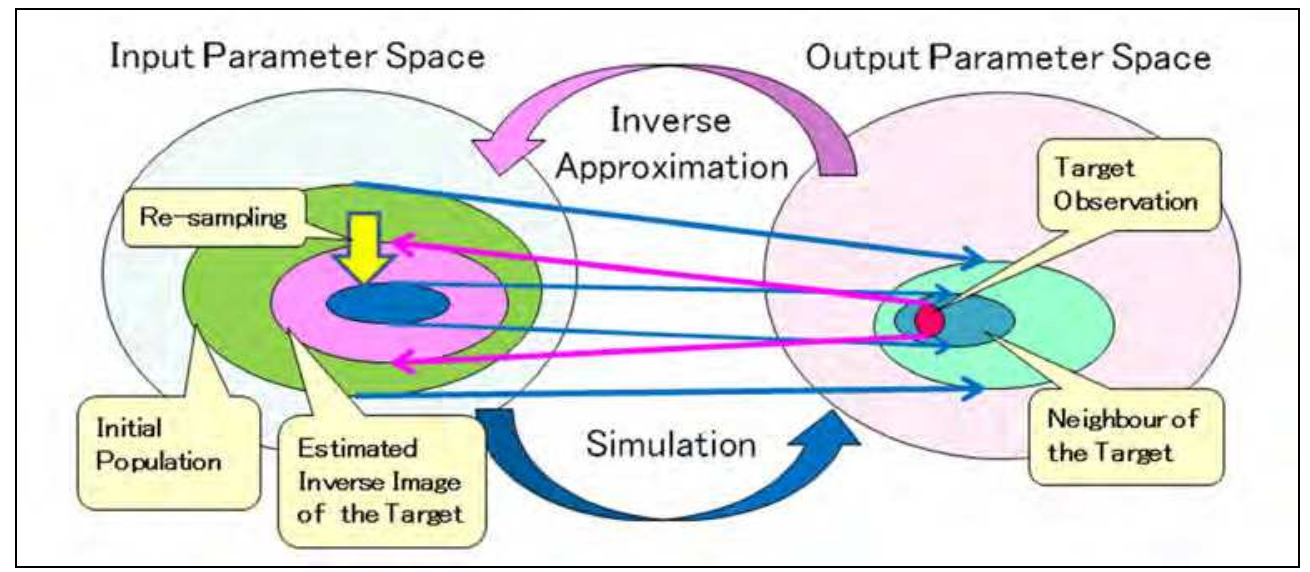

Fig. 6. Schematic View of Virtual Patient Population Convergence Virtual Patient Population Convergence estimates a virtual population mapping onto the neighbourhood of the target by iterating simulation and re-sampling of virtual patients. Intelligent sampling method is required to reduce total simulation costs for the convergence. 
First of all, each virtual patient should hold reasonable parameters from the viewpoints of physiology and pharmacokinetics. This requires that the search space should be restricted within or around the initial virtual patient population. Virtual patients with unrealistic parameters must be eliminated during the convergent process. Note that the search space is still wide enough since some pharmacokinetic parameters such as maximum velocity (Vmax) of enzymatic reaction range over the order of 10 to the power of 5 or 6 in an initial population.

Next, care must be taken for the handling of errors in the observed clinical data. The clinical data may have five or more percent of errors. Therefore, it is more important to preserve the diversity of virtual population as much as possible rather than to find a precise virtual patient reproducing the observed clinical data.

Lastly, convergence speed does matter. A mathematical model usually has dozens of unknown parameters. The virtual patient population convergence tries to find a "good" population by iteration of simulation and sampling by assigning new values to the unknown parameters of a virtual patient. Random sampling does not work well for this case due to the large search space. Sophisticated sampling algorithms must be developed to solve practical inverse problems of pharmacokinetics.

For example, Arikuma's irinotecan pharmacokinetic model has 61 input parameters and 10 output parameters with regards to irinotecan metabolism (Arikuma et al., 2008). Suppose that the goal of convergence is to find a virtual population whose error is within the ranges of $1 / 10$ of the target output on each output parameter. This may correspond to finding a rare event occurring one-tenth for each ten parameters, that is, once every 10 billion if we assume a uniform distribution.

Intelligent sampling such as Gibbs sampling and Markov Chain Monte Carlo (MCMC) methods are helpful to avoid redundant sampling. We accelerated the sampling performance of the MCMC method by using a support vector machine (SVM) to estimate the boundary of virtual patients reproducing the neighbour of the target output values. Intelligent sampling with regards to the boundary is much faster than the sampling with simulation, although some candidates inside the boundary may fall out of the neighbourhood of the target data due to the non-linear behaviour of simulation model.

\section{Case studies}

In order to demonstrate the effectiveness of the prototype system, we applied it to the irinotecan pharmacokinetic studies including multi-drug administration of irinotecan and ketokonazole, mutation effects of UGT1A1*28/*28 and the hepatic and renal excretion analysis of the bile-duct cancer patient with an external bile-drain. Irinotecan is an anticancer drug that is commonly used for colon and breast cancers (Mathijssen et al., 2001). Irinotecan is a prodrug of SN-38, anti-neoplastic topoisomerase I inhibitor, and is bioactivated by carboxyl esterase (CE). About $60 \%$ of irinotecan is excreted as unchanged drug from bile and kidney (Slatter et al., 2000). Irinotecan is also metabolized by CYP3A4 to form APC and NPC. NPC is further metabolized by CE to form SN-38. SN-38 undergoes glucuronate conjugation by UGT1A1 to form the inactive glucronide, SN-38G. 
Ketoconazole (KCZ) is an anti-fungal drug and a well-known inhibitor of CYP3A4. Ketoconazole undergoes extensive metabolism in the liver to form several metabolites (Whitehouse et al., 1994). About 2 to $4 \%$ of urinary radioactivity represents unchanged drug (Heel et al., 1982). It has been reported that the inhibition of CYP3A4 by ketoconazole influences the metabolism of irinotecan, which results in a 6\% SN-38 increase (Kehrer et al., 2002). In addition, it is known that the mutations on UGT1A1, UGT1A1*28/*28 which decreases the expression of UGT1A1 enzyme down to 30\%, has a strong relationship with some side effects of irinotecan (Sai et al., 2004; Ando et al., 2000).

Slatter et al. reported that the bile cancer patient with an external bile-drain showed completely different renal and hepatic metabolite excretion profiles from other cancer patients in their pharmacokinetic studies (Slatter et al., 2000). Why was the bile cancer patient so different from the other cancer patients? Which parameter caused the difference? This case is a typical example that requires personalized pharmacokinetics, in other words, the analysis of an outlier.

\subsection{Drug-drug Interaction between Irinotecan and ketoconazole}

The pathways of intravenously administered irinotecan and orally administered ketoconazole were inferred as aggregation of molecular events by the Pathway Object Constructor. The generated object included a metabolic pathway where irinotecan and its derivatives circulate through the veins, liver, bile, intestines, and portal vein, namely, the

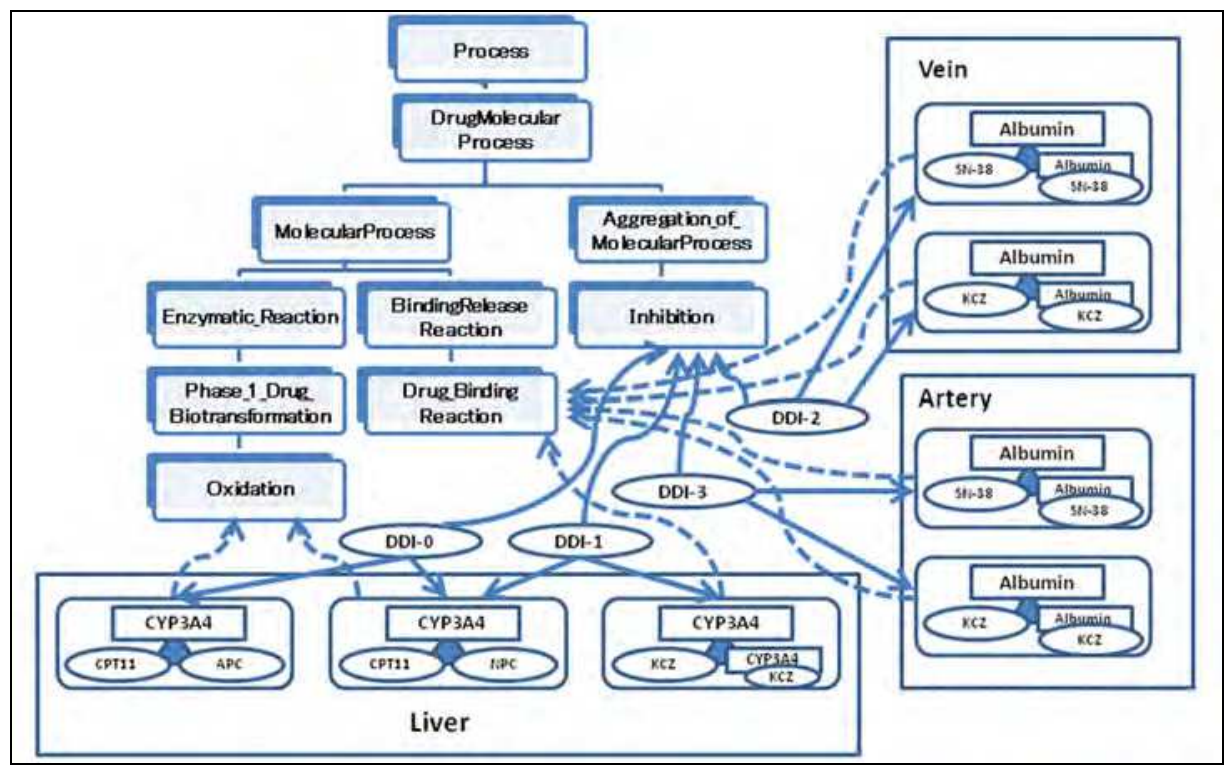

Fig. 7. Detected Drug-Drug Interactions mapped onto the Drug Interaction Ontology Four drug-drug interaction events are detected on the irinotecan and ketoconazole coadministrated metabolic pathway, which are mapped onto the Drug Interaction Ontology. Seven primitive reactions are involved in the events. Three of them and the rest are mapped onto the oxidation process and the drug_binding process, respectively. 
enterohepatic circulation, and are excreted through the kidneys or through the bile. These generated pathways were consistent with in vivo studies (Mathijssen et al., 2001). Interactions between intravenously administered irinotecan and orally administered ketoconazole were detected and asserted by the Drug Interaction Detector. The detected drug interactions and the hypothetic assertions are shown in Fig. 7. The assertion contains four drug interactions; two of them concern "drug binding reaction" to albumin in veins (ddi2) and arteries (ddi3), and the rest of them concern "oxidation" by CYP3A4 (ddi0 and ddi1). The detected drug interaction concerning CYP3A4 (ddi0 and ddi1) has been confirmed by the literature on in vivo studies (Kehrer et al., 2002).

We evaluated the effects of drug interactions concerning CYP3A4 quantitatively with numerical simulations. Intravenous drip infusion $\left(125 \mathrm{mg} / \mathrm{m}^{2}, 90 \mathrm{~min}\right)$ was assumed for irinotecan, and oral administration (200 mg) was assumed for ketoconazole. Two simulations were performed: sole administration of irinotecan for a patient having UGT1A1*1/*1 (wild type) and co-administration of irinotecan and ketoconazole for a patient having UGT1A $1{ }^{*} 1 /{ }^{*} 1$ (wild type). Fig. 8 (a) and (b) show the simulated concentration/time profiles of irinotecan, SN-38, APC, NPC, SN-38G in blood for the simulation of sole administration of irinotecan and co-administration of irinotecan and

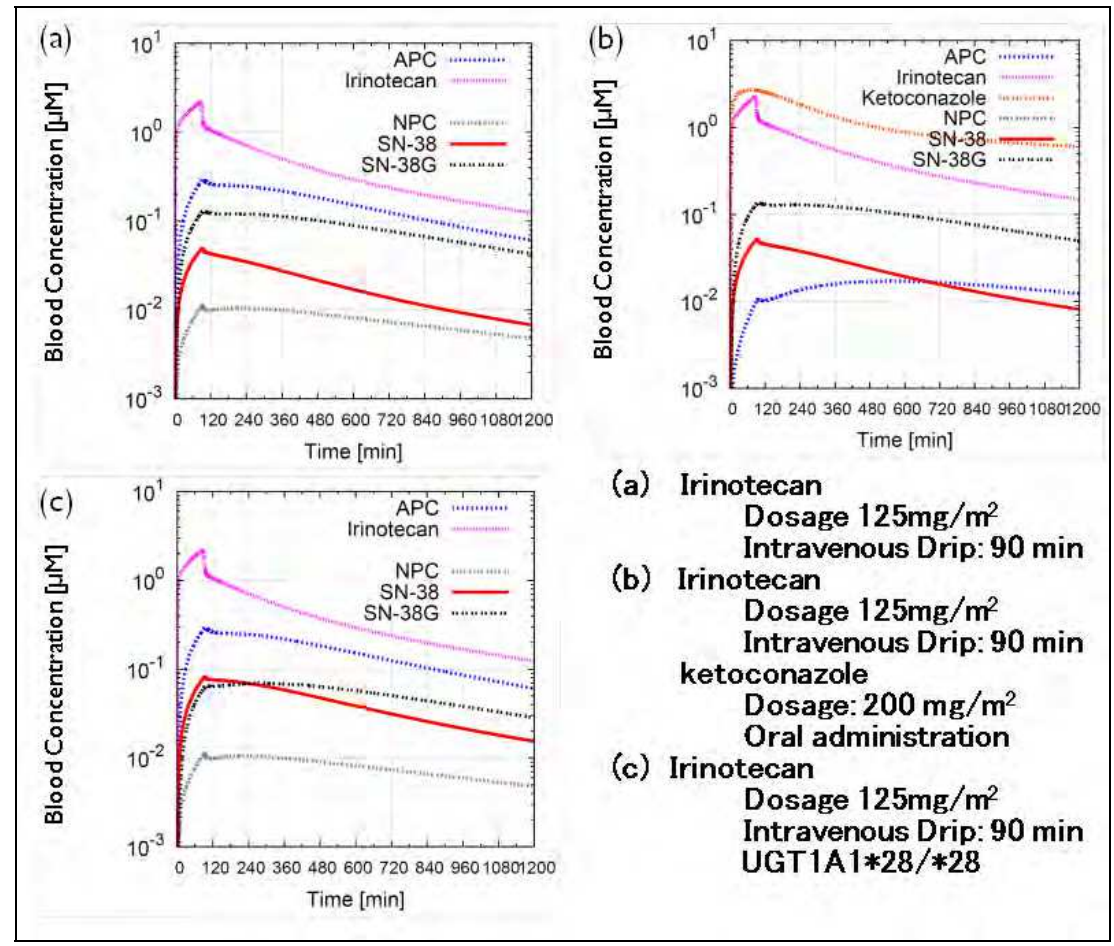

Fig. 8. Simulation Results of Time Course Concentration:
(a) Sole Irinotecan Administration (wild type)
(b)Multiple-administration of Irinotecan and Ketoconazole (wilde type)
(c) Sole Irinotecan Administration (UGT1A1*28/ 28 mutation) 
ketoconazole. By the ketoconazole administration, the area under the plasma concentrationtime curve (AUC) of APC and NPC were decreased to $48.1 \%$ and $35.3 \%$, respectively. The AUC of SN-38 was increased only to $108 \%$ by the ketoconazole administration. Similarly, the maximum drug concentration (Cmax) of APC and NPC were decreased to $25.6 \%$ and $20.2 \%$, respectively, whereas the Cmax of SN-38 was increased to $105 \%$ by the ketoconazole administration. This implies that effects of co-administration of ketoconazole and irinotecan are mild for the blood concentration of SN-38 in spite of inhibition of CYP3A4 in the drug metabolic pathway.

\subsection{Effects of UGT1A* $28 /{ }^{*} 28$ mutation}

It is reported that a patient with UGT1A1*28/*28 mutation decreased the expression of UGT1A1 down to $30 \%$ of patients with UGT1A1*1/*1 (wild type) in average. According to our quantitative evaluation with numerical simulation, the patient with UGT1A1*28/*28 mutation significantly increased the AUC and Cmax of SN-38: the AUC was increased to $208 \%$ and the Cmax was increased to $165 \%$ (Fig. 8 (c)). This implies that patients with UGT1A1*28/*28 may suffer severe side effects when the doses are the same as those for patients with UGT1A $1 * 1 /{ }^{*} 1$. These results agree with previously published experimental papers (Kehrer et al., 2002; Sai et al., 2004; Ando et al., 2000).

\subsection{Analysis of a bile-duct cancer patient with an external bile-drain}

In their pharmacokinetic study, Slatter et al. reported that the bile-duct cancer patient with an external bile-drain showed completely different renal and hepatic metabolite excretion profiles from other cancer patients (Slatter et al., 2000). Since the bile-duct cancer patient excreted her bile acid through an external bile-drain, it is possible to observe the hepatic clearances directly. As seen in Table 1, the ratio of hepatic and renal metabolite excretions is completely different between the bile-duct cancer patient and the other cancer patients; the ratio is almost the same for the bile-duct cancer patient while the ratio is 1-to-2 for the other cancer patients. Slatter et al. explained that the difference might result from the inhibition of canalicular multiple organic anion transporter (cMOAT/MRP2/ABCC2) in the bile-duct cancer patient. This is one of the typical examples that require the analysis of an outlier, that is, personalized pharmacokinetics.

\begin{tabular}{|c|c|c|c|c|}
\hline & \multicolumn{2}{|c|}{ Bile-duct Cancer Patient } & \multicolumn{2}{|c|}{$\begin{array}{c}\text { Other Cancer } \\
\text { Patients (N=7) }\end{array}$} \\
\hline & $\begin{array}{c}\text { Urine } \\
\text { Excretion }\end{array}$ & $\begin{array}{c}\text { Faeces } \\
\text { Excretion }\end{array}$ & $\begin{array}{c}\text { Urine } \\
\text { Excretion }\end{array}$ & $\begin{array}{c}\text { Faeces } \\
\text { Excretion }\end{array}$ \\
\hline CPT-11 & 21.8 & 24.7 & $22.4(5.5)$ & $32.3(4.5)$ \\
\hline APC & 7.7 & 5.6 & $2.2(1.5)$ & $8.3(3.0)$ \\
\hline SN-38G & 12.0 & 2.7 & $3.0(0.8)$ & $0.3(0.2)$ \\
\hline SN-38 & 0.9 & 3.2 & $0.4(0.1)$ & $8.2(2.5)$ \\
\hline NPC & 0.09 & 0.62 & $0.1(0.1)$ & $1.4(0.9)$ \\
\hline Total & 45.8 & 43.4 & $30.2(6.6)$ & $62.0(7.6)$ \\
\hline
\end{tabular}

Table 1. Percentages of Administrated Dose Excreted from Urine and Faeces in the Bile-duct Cancer Patient and the Average of Other Cancer Patients summarized from the Pharmacokinetic Study reported by (Slatter et al. 2000). 
Bile acid excretion and faeces excretion are added for the bile-duct cancer patient. The total percentage does not recover all administrated dose due to the experimental limitations and drug metabolisms other than the CPT-11, APC, SN-38G, SN-38, and NPC.

Arikuma et al. have developed an irinotecan metabolic pathway model with five major irinotecan metabolites: CPT-11(irinotecan), APC, NPC, SN-38 and SN-38G (Arikuma et al., 2008). Since those components occupy about 90 percent of irinotecan metabolites, it is sufficient to consider the dynamics of blood concentration of the five compounds with renal and hepatic excretions as a pharmacokinetic model. The challenge for personalized pharmacokinetics is whether Arikuma's model can reproduce the bile-duct patient data or not.

Figure 9 shows the set of virtual patients whose simulation results fall into the neighbourhood of the bile-duct patient within a 5 percent error margin in hepatic and renal excretions of CPT-11, APC, NPC, SN-38 and SN-38G after virtual patient population convergence. The distribution shows clear dependency in hepatic and renal excretions in CPT-11, but no such dependencies in other components.

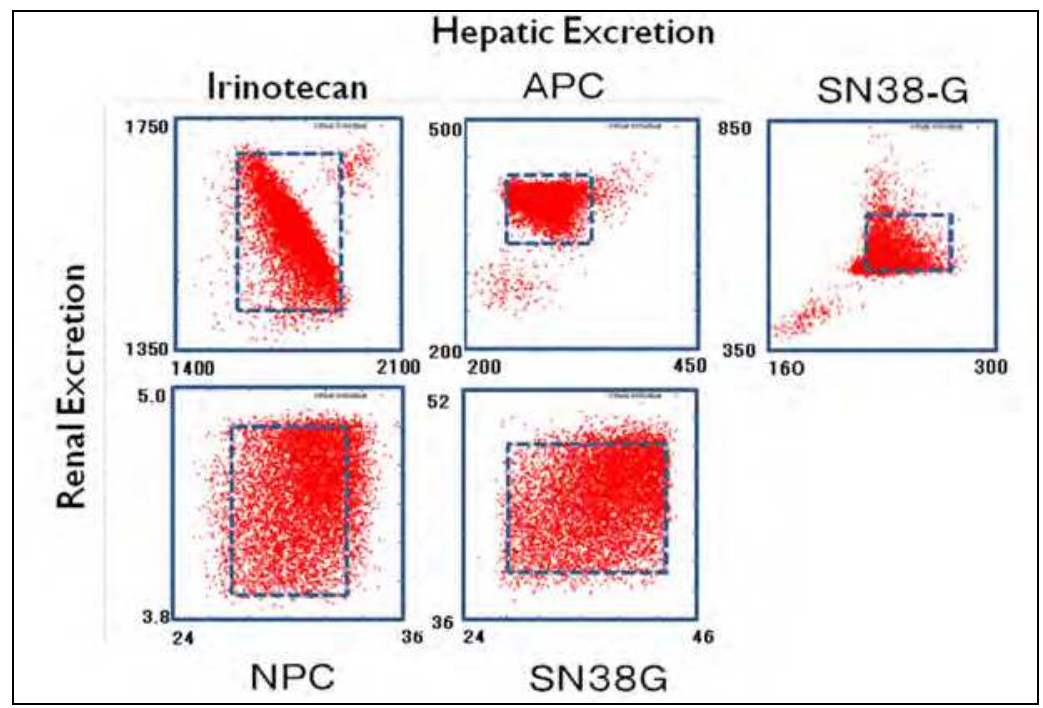

Fig. 9. Distribution of Virtual Patient Population reproducing the Hepatic and Renal Irinotecan Metabolite Excretions of the Bile-duct Cancer Patient Horizontal and vertical axes represent hepatic and renal excretions, respectively. The centre of each square in the graphs is the target excretion of the bile-duct cancer patient estimated from the pharmacokinetic study reported by Slatter et al. 2000. Each dot represents a virtual patient whoes hepatic and renal excretions are within the five percent error ranges on the pharmacokinetic simulation.

In the case of an undetermined inverse problem, what we can obtain is just a multi-set of solution candidates. We cannot determine the true solution because of the lack of conditions. However, it would be reasonable to expect that the solution candidates may have common properties reproducing the target data in the inverse problem. Figure 10 shows the averages and distributions of the hepatic clearances converged to metabolic 
excretions of the bile-duct cancer patient and the averages of other cancer patients. Intriguingly, there is a clear down-regulation in APC, SN-38, and SN-38G between the two, suggesting coincidence with the pharmacogenetic study of ABCC2 (Innocenti et al., 2009).

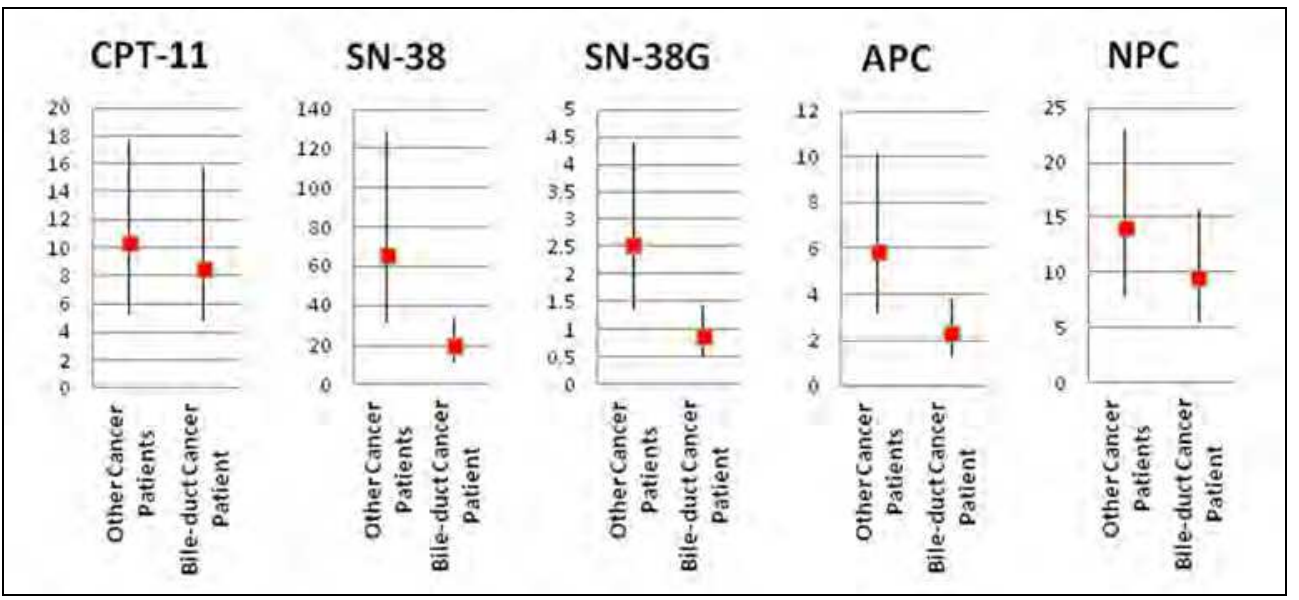

Fig. 10. Comparison of the bile-duct cancer patient and the other cancer patients with regards to the Average and Distribution of Hepatic Clearances in Virtual Patient Population after Convergence

Horizontal axis represents estimated hepatic clearances in numerical simulation (unit $\left.1 / \mathrm{h} / \mathrm{m}^{2}\right)$.

\section{Discussion}

Our study on personalized pharmacokinetics is still in its infancy. Ontology and mathematical simulation are powerful information technologies but need more research for practical use, especially from the viewpoints of knowledge representation and unknown parameter estimation. In this section, we discuss these issues.

\subsection{Knowledge representation and inference}

It is widely known that there is a trade-off between the amount of knowledge base and deepness of inference in knowledge representation, that is, shallow inference on huge knowledge base and deep inference on small knowledge base. Both approaches have their own merits. Therefore, it is important to choose the right size of knowledge base and the right level of inference in knowledge representation.

As for biomedical ontologies, OWL becomes one of de facto standards, especially in the world of Semantic Web, the network of knowledge with inference in the Internet (Timizi et al, 2011). It adopts a unique representation form of knowledge with UNICODE, URI (Uniform Resource Identifier) and RDF (Resource Description Form), and provides an inference engine, named reasoning based on description logic (Zhang et al., 2006).

We adopted OWL-DL for the implementation of the Drug Interaction Ontology to make it consistent with reference ontologies such as Foundation of Model of Anatomy (FMA) 
(Rosse\&Mejino, 2003) and Unified Medical Language System (UMLS) (Bodenreider, 2004). The reference ontologies enable us to focus on the development of a control vocabulary specific to pharmacokinetics while allowing the usage of general technical terms as defined in the reference ontologies. In addition, the OWL reasoner helps indentify unsatisfiable classes and consistency checking in Ontology (Zhang et al., 2006).

However, it is apparent that OWL-DL is not suitable for the detection of drug-drug interactions and the generation of metabolic pathways. We strongly believe that inference programs on ontology should not be restricted to the level of reasoning. Therefore, we developed our inference programs in Prolog while using OWL-DL for consistency checking of the Drug Interaction Ontology.

The inference programs may infer new assertions that cannot be deduced from the original ontology in reasoning. In such a case, it is impossible for computers to validate the assertions automatically. In order to compensate for the lack of automatic validation, we introduced hypothetical links that map the assertions on the Drug Interaction Ontology. The hypothetical links give useful background information for human beings to validate the assertions. For example, in the case of the drug-drug interaction detection of irinotecan and ketoconazole, four drug-drug interaction events (from ddi0 to ddi3 in Fig. 6) were found by our inference program. Mapping the events onto the process vocabulary hierarchy enables us to interpret ddi0 and ddi1 as enzymatic inhibition of CYP3A4 while ddi2 and ddi3 are binding confliction on albumin.

\subsection{Distribution estimation}

Mathematical models expressed by ordinal differential equations define the mapping from parameter space to accumulation data space when integrating the equations from zero to a specific time point. The mapping is mostly nonlinear, and multiple sets of parameters may reproduce the same accumulation data. The virtual patient population convergence is a technique to estimate the distribution of virtual patients reproducing the same or similar accumulation data when the initial population is given in an inverse problem.

From the viewpoint of parameter distribution estimation algorithms, the virtual patient population convergence raises several issues such as initial population dependency and early convergence to local minima as well as convergence performance. As for the convergence performance, we have developed a very fast deterministic algorithm, the details of which will be published elsewhere (Aoki et al., 2011). Further studies should be carried out for the rest.

Another important issue related to the virtual patient population convergence is the interpretation of solution space, that is, the obtained population after convergence. Mathematical models often have parameter-parameter dependencies that compensate for the effect of certain parameters by means of adjusting other parameters to reproduce the same output (Azuma et al., 2007). This suggests focusing on the analysis of parameter diversities of the solution space, rather than the analysis of an individual virtual patient of the solution space. In case of the bile-duct cancer patient of irinotecan pharmacokinetics, the virtual patients in solution space falls into specific parameter ranges on hepatic clearances. However, care must be taken when the ranges seem to be strange from the viewpoint of biology and medical science. In such a case, the specific ranges may result from a deficiency of the model to explain the behavior of the outlier. 


\section{Conclusions}

The effectiveness of personalized pharmacokinetic is demonstrated by the in silico analysis of an irinotecan pharmacokinetic study with the Drug Interaction Ontology and automatic drug metabolic pathway generation followed by numerical simulation.

As for drug-drug interaction detection, the prototype system detected four drug interactions for an irinotecan plus ketoconazole regimen. Two of them concerned cytochrome p450 (CYP3A4) and were consistent with known drug interactions. The numerical simulation indicates that the effect of the drug-drug interactions is mild for the increase of SN-38 blood concentration although APC and NPC blood concentrations are reduced considerably.

We then quantitatively examined the effect of genetic variation UGT1A1*28/*28 using numerical simulations. The genetic variation on UGT1A1 showed a two-fold increase of SN38's AUC as suggested by the literature (Gagne et al., 2002).

Finally, we analyzed the pharmacokinetic parameters reproducing the bile-duct cancer patient with an external bile-drain in terms of hepatic and renal metabolic excretions of CPT-11, APC, NPC, SN-38 and SN-38G. The obtained virtual patients suggests that the difference of hepatic clearances in APC, SN-38 and SN-38G may be the major reason that causes the clinical differences between the bile-duct cancer patient and the other cancer patients in irinotecan pharmacokinetics.

\section{Acknowledgments}

The author expresses his thanks to Mr. Takeshi Arikuma and Mr. Takashi Watanabe for the development of the prototype system including the Drug Interaction Ontology; the inference programs of automatic generation of pathways and models, and the detection of drug-drug interactions, the numerical simulation engine with virtual patient population convergence facility.

\section{References}

Aoki, Y.; Hayami, K.; Hans, DS. \& Konagaya, A. (2011). Cluster Newton Method for Sampling Multiple Solutions of an Underdetermined Inverse Problem: Parameter Identification for Pharmacokinetics, NII Technical Report (NII-2011-002E)

Ando, Y.; Saka, H.; Ando, M.; Sawa, T.; Muro, K.; Ueoka, H.; Yokoyama, A.; Saitoh, S.; Shimokata, K. \& Hasegawa, Y. (2000). Polymorphisms of UDP-Glucuronosy ltransferase Gene and Irinotecan Toxicity: A Pharmacogenetic Analysis, Cancer Research, Vol. 60, pp.6921-6926

Angrist, M. (2007). Here is a Human Being at the Dawn of Personal Genomics, Harper

Arikuma, T.; Yoshikawa, S.; Watanabe, K.; Matsumura, K. \& Konagaya, A. (2008). Drug Interaction Prediction using Ontology-driven Hypothetical Assertion Framework for Pathway Generation followed by Numerical Simulation, BMC Bioinformatics, vol. 9 (Suppl 6), S11

Azuma, R.; Umetsu, R.; Ohki, S.; Konishi, F.; Yoshikawa, S., Matsumura, K. \& Konagaya, A. (2007). Discovering Dynamic Characteristics of Biochemical Pathways using Geometric Patterns among Parameter-Parameter Dependencies in Differential Equations, New Generation Computing, Vol.25, pp.425-441 
Baker, CJ. \& Cheung, KH. (2007) Semantic Web: Revolutionizing Knowledge Discovery in the Life Sciences, Springer

Berners-Lee, T. \& Hendler J. (2001). The Semantic Web, Nature, Vol.410, No.6832, pp.10231024

Bodenreider, O. (2004). The Unified Medical Language System (UMLS): Integrating Biomedical Terminology, Nucleic Acids Research, Vol.32, D267-D270

Ceusters,W. \& Smith,B. (2010). A Unified Framework for Biomedical Terminologies and Ontologies, Studies in Health Technology and Informatics, Vol.160(Pt 2),pp.1050-1054

Chien, JY. ; Lucksiri, A.: Charles, S.; Ernest, I.; Gorski, JC.; Wrighton, SA. \& Hall SD. (2006). Stochastic Prediction of CYP3A-Mediated Inhibition of Midazolam Clearance by Ketoconazole, Drug Metab Dispos, Vol.34, No.7, pp.1208-1219

Collins, FS.; Green, ED.; Guttmacher, AE. \& Guyer, MS. (2003). A Vision for the Future of Genomic Research, Nature, vol. 422, pp.835-847

Gabrielsson, J.; Dolgos, H.; Gillberg, PG.; Bredberg, U.; Benthem, B. \& Duker, G. (2009). Early Integration of Pharmacokinetic and Dynamic Reasoning is Essential for Optimal Development of Lead Compounds: Strategic Consideration, Drug Discovery Today, Vol.14, No.7, pp.358-372

Gagne, JF.; Montminy, V.; Belanger, P.; Journault, K.; Gaucher, G. \& Guillemette C. (2002). Common Human UGT1A Polymorphisms and the Altered Metabolism of Irinotecan Active Metabolite 7-ethyl-10-hydroxycamptothecin (SN-38), Molecular Pharmacology, Vol.62, No.3, pp.608-617

Grenon, P. \& Smith, B. (2004a). SNAP and SPAN: Towards Dynamic Spatial Ontology. Spatial Cognition and Computation, Vol.1, pp.69-103

Grenon, P.; Smith, B. \& Goldberg, L. (2004b). Biodynamic Ontology:Applying BFO in the Biomedical Domain, In: Ontologies in Medicine Amsterdam, IOS Press, pp.20-38

Heel, R.; Brogden, RA.; Carmine, PM.; Speight, T. \& Avery, G. (1982). Ketoconazole: A review of its Therapeutic Efficacy in Superficial and Systemic Funfal Infections, Drugs, Vol.23, pp.1-36

Innocenti,F.; Kroetz, DL.; Schuetz, E.; Dolan, ME.; Ramirez, J.; Relling, M.; Chen,P.; Das,S.; Rosner,GL. \& Ratain,MJ. (2009). Comprehensive Pharmacogenetic Analysis of Irinotecan Neutropenia and Pharmacokinetics, Journal of Clinical Oncology, Vol.27, No.16, pp.2601-2614

Kanehisa, M. \& Goto, S. (2000). KEGG: Kyoto Encyclopedia of Genes and Genomes, Nucleic Acids Research, Vol.28, pp.27-30

Kehrer, DF.; Mathijssen, RH.; Verweij, J; de Bruijn, P. \& Sparreboom, A. (2002). Modulation of Irinotecan Metabolism by Ketoconazole, Journal of Clinical Oncology, Vol.20, No.14, pp.3122-3129

Konagaya, A. (2006a). OBIGrid: Towards the Ba for Sharing Resources, Services and Knowledge for Bioinformatics, Proceedings of the 4th International Workshop on Biomedical Computations on the Grid (BioGrid' 06), Singapore

Konagaya, A. (2006b). Trends in Life Science Grid: from Computing Grid to Knowledge Grid, BMC Bioinformatics, Vol.7 (Suppl 5):S10

Mathijssen, RHJ.; van Alphen, RJ.; Verweij ,J.; Loos, WJ.; Nooter, K.; Stoter, G. \& Sparreboom, A. (2001). Clinical Pharmacokinetics and Metabolism of Irinotecan (CPT-11), Clinical Cancer Research, Vol.7, pp.2182-2194

Mitchelson, KR. (Ed.) (2007). New High Throughput Technologies for DNA Sequencing and Genomics, Elsevier, UK 
Okuda, H.; Ogura, K.; Kato, A.; Takubo, H. \& Watanabe, T. (1998). A Possible Mechanism of Eighteen Patient Deaths Caused by Interactions of Sorivudine, a New Antiviral Drug, with Oral 5-fluorouracil Prodrugs. J Pharmacol Exp Ther, Vol. 287, No.2, pp.791-799

Pirmohamed, M. (2011). Pharmacogenetics: past, present and future, Drug Discovery Today, Aug. 22nd,Epub ahead of print

Rosse, C. \& Mejino, JLV. (2003). A Reference Ontology for Biomedical Informatics: the Foundational Model of Anatomy, Journal of Biomedical Informatics, Vol.36, pp.478-500

Sai, K.; Saeki, M.; Saito, Y.; Ozawa, S.; Katori, N.; Jinno, H.; Hasegawa, R.; Kaniwa, N.; Sawada, J.; Komamura, K., Ueno, K.; Kamakura, S.; Kitakaze, M., Kitamura, Y.; Kamatani, N.; Minami, H; Ohtsu, A; Shirao, K.; Yoshida, T. \& Saijo, N. (2004). UGT1A1 Haplotypes Associated with Reduced Glucuronidation and Increased Serum Bilirubin in Irinotecanadministered Japanese Patients with Cancer. Clin Pharmacol, Ther, Vol. 75, pp.501-515

Slatter, JG.; Schaaf, LJ.; Sams, JP.; Feenstra, KL.; Johnson, MG.; Bombardt, PA.; Cathcart, KS; Verburg, MT.; Pearson, LK.; Compton, LD.; Miller, LL.; Baker, DS.; Pesheck, CV.; Raymond, S. \& Lord, I. (2000). Pharmacokinetics, Metabolism, and Excretion of Irinotecan (CPT-11) Following I.V. Infusion of [14C]CPT-11 in Cancer Patients, Drug Metabolism and Disposition, Vol.28, No.4, pp.423-433

Timizi,SH.; Aitken,S.; Moreira, DA.; Mungall, C.; Seueda, J.; Shah, NH. \& Miranker, DP. (2011). Mapping between the OBO and OWL Ontology Languages, Journal of Biomedical Semantics 2011, Vol.2 (Suppl 1):S3

Tsukamoto, Y.; Kato Y; Ura. M.; Horii, I.; Ishitsuka, H.; Kusuhara, H. \& Sugiyama, Y. (2001). A Physiologically based Pharmacokinetic Analysis of Capecitabine, a Triple Prodrug of 5-FU, in Humans, the Mechanism for Tumor-selective Accumulation of 5-FU, Pharm Res, Vol.18, No.8, pp.1190-1202

Tukey, RH.; Strassburg, CP. \& Mackenzie, PI. (2002). Pharmacogenomics of Human UDPGlucuronosyltransferases and Irinotecan Toxicity, Molecular Pharmacology, Vol.62, No.3, pp.446-450

Vossen, M.; Sevestre, M.; Niederalt. C.; Jang, IJ.; Willmann. S. \& Edginton, AN. (2007). Dynamically Simulating the Interaction of Midazolam and the CYP3A4 Inhibitor Itraconazole using Individual Coupled Whole-Body Physiologically-based Pharmacokinetic (WBPBPK) Models. Theoretical Biology and Medical Modelling, Vol. 4, No. 13

Whitehouse, LW.; Menzies, A.; Dawson, B.; Cyr, TD.; By AW.; Black, DB. \& Zamecnik, J. (1994). Mouse Hepatic Metabolites of Ketoconazole: Isolation and Structure Elucidation, J Pharm Biomed Anal 1994, Vol. 2, No.11, pp.1425-1441

Willmann, S.; Hohn, K.; Edginton, A.; Sevestre, M.; Solodenko, J.; Weiss, W.; Lippert, J. \& Schmitt ,W. (2007). Development of a Physiology-based Whole-Body Population Model for Assessing the Influence of Individual Variability on the Pharmacokinetics of Drugs, Pharmacokinet Pharmacodyn, Vol. 34, No.3, pp.401-431

Yoshikawa, S.; Kenji, S. \& Konagaya, A. (2004). Drug Interaction Ontology (DIO) for Inferences of Possible Drug-Drug Interactions, Medinfo, Vol.11, pp.454-458

Zhang, S.; Bodenreider, O. \& Golbreich, C. (2006). Experiences in Reasoning with the Foundational Model of Anatomy in OWL DL, Pacific Symposium on Biocomputing 2006, pp.200-211 


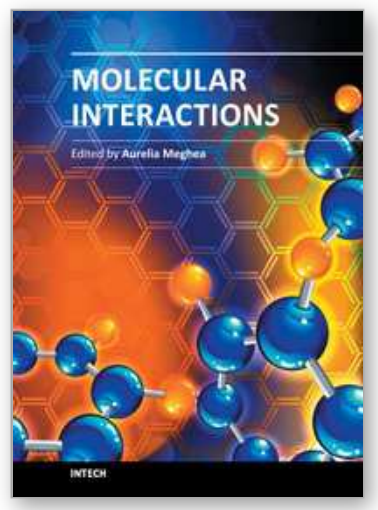

\author{
Molecular Interactions \\ Edited by Prof. Aurelia Meghea
}

ISBN 978-953-51-0079-9

Hard cover, 400 pages

Publisher InTech

Published online 29, February, 2012

Published in print edition February, 2012

In a classical approach materials science is mainly dealing with interatomic interactions within molecules, without paying much interest on weak intermolecular interactions. However, the variety of structures actually is the result of weak ordering because of noncovalent interactions. Indeed, for self-assembly to be possible in soft materials, it is evident that forces between molecules must be much weaker than covalent bonds between the atoms of a molecule. The weak intermolecular interactions responsible for molecular ordering in soft materials include hydrogen bonds, coordination bonds in ligands and complexes, ionic and dipolar interactions, van der Waals forces, and hydrophobic interactions. Recent evolutions in nanosciences and nanotechnologies provide strong arguments to support the opportunity and importance of the topics approached in this book, the fundamental and applicative aspects related to molecular interactions being of large interest in both research and innovative environments. We expect this book to have a strong impact at various education and research training levels, for young and experienced researchers from both academia and industry.

\title{
How to reference
}

In order to correctly reference this scholarly work, feel free to copy and paste the following:

Akihiko Konagaya (2012). Towards an In Silico Approach to Personalized Pharmacokinetics, Molecular Interactions, Prof. Aurelia Meghea (Ed.), ISBN: 978-953-51-0079-9, InTech, Available from:

http://www.intechopen.com/books/molecular-interactions/towards-an-in-silico-approach-to-personalizedpharmacokinetics

\section{INTECH}

open science | open minds

\section{InTech Europe}

University Campus STeP Ri

Slavka Krautzeka 83/A

51000 Rijeka, Croatia

Phone: +385 (51) 770447

Fax: +385 (51) 686166

www.intechopen.com

\section{InTech China}

Unit 405, Office Block, Hotel Equatorial Shanghai

No.65, Yan An Road (West), Shanghai, 200040, China

中国上海市延安西路65号上海国际贵都大饭店办公楼405单元

Phone: +86-21-62489820

Fax: +86-21-62489821 
(C) 2012 The Author(s). Licensee IntechOpen. This is an open access article distributed under the terms of the Creative Commons Attribution 3.0 License, which permits unrestricted use, distribution, and reproduction in any medium, provided the original work is properly cited. 